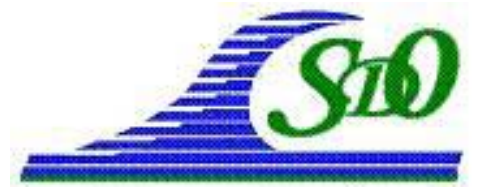

XI ${ }^{\text {èmes }}$ Journées Nationales Génie Côtier - Génie Civil

Les Sables d'Olonne, 22-25 juin 2010

DOI:10.5150/jngcgc.2010.029-F @ Editions Paralia CFL

disponible en ligne - http://www.paralia.fr - available online

\title{
Modélisation conceptuelle d'un littoral à double barre festonnée en milieu microtidal
}

\author{
Pierre FERRER ${ }^{1}$, Raphael CERTAIN ${ }^{1}$, \\ Jean-Paul BARUSSEAU ${ }^{1}$, Mathieu GERVAIS ${ }^{1,2}$
}

1. IMAGES, Université de Perpignan Via Domitia, 52 Av. Paul Alduy, 66860 Perpignan Cedex, France.

pierre.ferrer@univ-perp.fr

2. B.R.G.M. section Languedoc-Roussillon, 1039, rue de Pinville, 34000 Montpellier, France.

\section{Résumé :}

Les systèmes de barres sédimentaires ont été largement observés en milieux méso- et macrotidaux. Leur étude en milieu microtidal est moins fréquente. La partie sud du littoral du golfe du Lion (mer Méditerranée) et son système à deux barres festonnées ont été observés mensuellement durant un suivi pluriannuel de 3 ans (2005-2008) et annuellement à partir de 2000. L'objet de cette communication est de proposer un modèle conceptuel d'évolution basé sur la morphodynamique de ce système microtidal, à partir des suivis topo-bathymétriques et des mesures hydrodynamiques. Ce modèle est basé sur différentes séquences évolutives observées. Il est ensuite comparé avec d'autres observations faites sur d'autres sites micro-, méso- et macrotidaux.

Mots-clés :

Morphodynamique - Plage - Barres festonnées - Modèle conceptuel - Microtidal

\section{Introduction}

La plupart des côtes sableuses présentent des systèmes de barres. La principale classification des types de barres est proposée par SHORT \& AAGAARD (1993). Les travaux de BRANDER (1999) viennent compléter cette classification en additionnant plusieurs états intermédiaires de plage. Au cours de ce travail, nous nous focaliserons sur les barres de type festonné appelées "Rhythmic Bar and Beach" (RBB) dans cette classification. L'état RBB correspond à un état de plage intermédiaire.

L'évolution morphologique de ces motifs a été observée en différents lieux de par le monde en utilisant différentes techniques (VAN ENCKEVORT et al., 2004). D'une manière générale, pour un système double de barres, le point de départ de leur formation est une barre externe parallèle à la côte (VAN ENCKEVORT et al., 2004 ; CERTAIN \& BARUSSEAU, 2005 ; CASTELLE et al., 2007), la configuration de la barre interne peut être variée. Durant un épisode de tempête, le système double de barres migre vers le large. Quand les conditions hydrodynamiques deviennent moins énergétiques, la barre externe forme dans un premier temps une "longshore bar-trough morphology" 
(LBT) (CASTELLE et al., 2007) ou une "meandering bar" (GOLDSMITH et al., 1982) avant d'évoluer en une barre festonnée (RBB) ; la barre interne peut également présenter plusieurs configurations excluant LBT. Après un évènement de tempête, le système à deux barres migre vers la côte et la rythmicité des motifs augmente (CASTELLE et al., 2007). Si la barre interne est festonnée, elle peut s'attacher à la plage par l'intermédiaire de ses cornes sous la forme de barres transverses ou "Transverse Bar and Rip" (TBR) sous des conditions de houle de faible énergie (DE MELO APOLUCENO, 2003). Quand un évènement de forte énergie se reproduit, avec une hauteur significative supérieure à $2 \mathrm{~m}$ et une incidence oblique, la barre interne peut prendre une configuration en TBR oblique comme sur le site d'Hahoterim Beach (GOLDSMITH et al., 1982) ou du Truc Vert (CASTELLE et al., 2007), et pour les plages de Duck ou de Miyasaki pour des systèmes à barre unique (VAN ENCKEVORT et al., 2004). En présence de barres transverses, une petite barre d'extension longitudinale ou "rip head bar" peut apparaitre suivant la forme du courant d'arrachement (BRANDER, 1999). Sous des conditions de forte énergie, la barre externe festonnée se perce et migre à la côte. Sur la côte Aquitaine, la barre interne en configuration TBR peut se maintenir lors d'évènements avec des hauteurs significatives supérieures à 5 m (CASTELLE et al., 2007). Après le pic de tempête, la barre interne TBR peut se réorganiser en système de barres/baines ou "Low Tide Terrace" (LTT) si les conditions sont de très faible énergie pendant une durée assez longue (BRANDER, 1999 ; DE MELO APOLUCENO, 2003 ; CASTELLE et al., 2007).

Connaissant ce modèle général, l'objectif de ce travail est de proposer un modèle conceptuel pour un système à deux barres festonnées en milieu microtidal incluant également la plage émergée. Nous espérons ainsi pouvoir compléter le modèle général en y incorporant de nouveaux états intermédiaires et affiner la connaissance des paramètres de contrôle. Les séquences d'évolution ainsi décrites seront ensuite incorporées et discutées par rapport à la classification de SHORT \& AAGAARD (1993). L'application de ce modèle sera comparée aux observations faites sur d'autres sites micro-, méso- et macrotidaux.

\section{Zone d'étude}

Leucate-Plage se situe en Languedoc-Roussillon, dans la partie sud des côtes méditerranéennes françaises (figure 1). Le choix de ce site a été fait en fonction des connaissances antérieures et du motif festonné prépondérant ici. La plage est orientée nord-sud, limitée au nord par les falaises de Leucate et au sud par le grau des ostréiculteurs. Cet environnement de type microtidal est caractérisé par une marée moyenne annuelle de $0,17 \mathrm{~m}$, il est dominé par des vagues de hauteur significative moyenne annuelle de 0,62 m et une période moyenne autour de 4,12 s (source DREAL Languedoc-Roussillon) ne mettant pas en évidence la grande variabilité saisonnière (beaucoup plus d'évènements de tempêtes en automne et hiver). Durant les épisodes de 


\section{XI ìmes Journées Nationales Génie Côtier-Génie Civil}

Les Sables d'Olonne, 22-25 juin 2010

tempêtes, la hauteur des vagues peut atteindre $9 \mathrm{~m}$ (au large) pour une période maximale de 9,2 $\mathrm{s}$. Morphologiquement, le système est caractérisé par la présence de deux barres festonnées et une pente moyenne de la plage de 1,5\%. La barre interne est localisée entre 100 et $200 \mathrm{~m}$ de la côte et possède une longueur d'onde moyenne de 300 $\mathrm{m}$ alors que la barre externe est localisée entre 400 et $500 \mathrm{~m}$ de la côte et possède une longueur d'onde moyenne de $600 \mathrm{~m}$ (BARUSSEAU \& SAINT-GUILY, 1981; CERTAIN, 2002 ; FERRER et al., 2009).

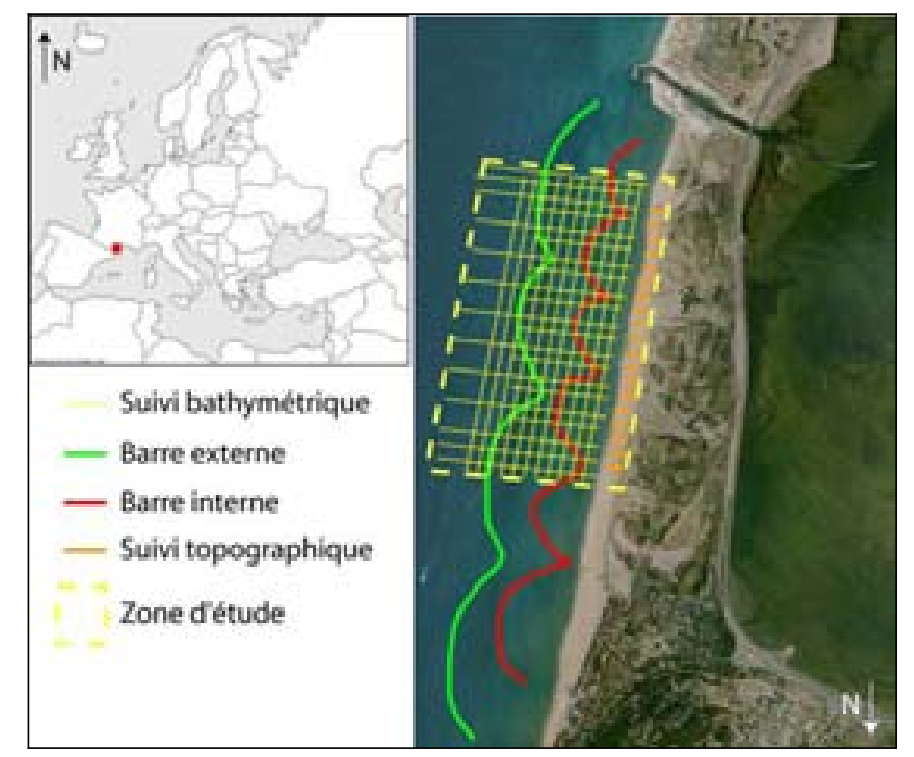

Figure 1. Localisation du site d'étude sur la côte méditerranéenne, montrant un système double de barres festonnées, ainsi que le plan de positionnement des différents suivis (bathymétriques, topographiques et trait de côte).

\section{Méthodologie}

\subsection{Les données morphologiques}

Les données ont été acquises mensuellement d'avril 2006 à septembre 2008. Cette base de données est complétée par plusieurs levés réalisés durant les étés 2000, 2001, 2002 et un dernier postérieur aux évènements de tempêtes en 2009. Elle est donc constituée par 28 cartes bathymétriques réalisées post-tempêtes à partir de 11 lignes longitudinales et de 21 lignes transversales. L'espacement transversal entre les profils est de $100 \mathrm{~m}$, celui longitudinal est de $100 \mathrm{~m}$ au niveau de la barre externe et de $50 \mathrm{~m}$ au niveau de la barre interne. Le suivi topographique a été réalisé à partir de 20 profils transversaux, espacés chacun de $50 \mathrm{~m}$. Toutes ces données ont été compilées afin de réaliser des cartes topobathymétriques sous Surfer®. Chaque carte est ensuite comparée avec la suivante, le z de référence est celui de la première carte. 


\subsection{Les données hydrodynamiques}

Les données hydrodynamiques ont été obtenues grâce à une bouée Datawell localisée aux coordonnées suivantes $42^{\circ} 55,000^{\prime} \mathrm{N}$ et $3^{\circ} 07,500^{\prime} \mathrm{E}$, juste au large du site d'étude et ancrée sur une profondeur de $40 \mathrm{~m}$ (DRE Languedoc-Roussillon) durant tout le suivi mensuel. Une autre bouée directionnelle, la bouée POEM, a également été utilisée afin de compléter la base de données. Ponctuellement, une campagne intensive de mesures hydrodynamiques a été réalisée en janvier-février 2007 dans le but de caractériser la circulation hydrodynamique au niveau d'une zone s'étendant d'un feston externe jusqu'à la côte.

\section{Classification des différents états de plage observés}

Au cours de ce suivi, les positions géographiques des barres externe et interne et en particulier de leurs cornes n'ont pas subi de fortes évolutions par rapport aux mesures antérieures (BARUSSEAU \& SAINT-GUILY, 1981 ; CERTAIN, 2002 ; FERRER et $a l .$, 2009) (figure 2). La barre externe présente ainsi un motif permanent en festons, même si les hauteurs de ses cornes ont pu varier en altitude; alors que la barre interne prend plusieurs motifs durant le suivi. Plusieurs états se sont en effet succédés (RBB, TBR regular, TBR oblique, TBR-LTT) sous l'influence du forçage hydrodynamique (hauteur, période, direction notamment) qui a pu être caractérisé pour chaque évènement. Seul un exemple est présenté ici (figure 3). De plus, de nouveaux états ont également été observés (TBR oblique rompu, RBB rompu).

\section{Modèle conceptuel morphodynamique pour le site de Leucate-Plage}

Les nombreuses transitions observées durant le suivi ainsi que le forçage associé ont permis d'élaborer un modèle conceptuel d'évolution pour les barres du littoral de Leucate-Plage (figure 4). Ce modèle présente 8 états de plage observés, tous de type intermédiaire (WRIGHT \& SHORT, 1984). Dans ce modèle, deux types de relation sont indiquées, les flèches en trait continu décrivent des transitions observées (Cas 1, 2, 3, 4, 5 et 6) alors que celles en trait pointillé indiquent des transitions possibles. Ce modèle est détaillé pour un système à deux barres, dans lequel la barre externe conserve le même motif RBB durant tout le suivi. Ce modèle décrit donc des transitions au niveau de la barre interne.

Le point de départ du modèle est le motif RBB. Cette configuration est en effet, la plus observée sur ce site depuis 1961 (RIVIERE et al., 1961 ; BARUSSEAU \& SAINTGUILY, 1981 ; CERTAIN, 2002...). Les cornes de la barre interne constituent des points hauts à position peu variable durant le suivi. Les séquences décrites ci-dessous sont le fruit de l'influence des paramètres de la houle qui contrôle le plus ce système: hauteur significative et incidence.

Quand le système est soumis à une houle frontale de hauteur significative modérée, la barre interne migre à la côte. La liaison à la côte des points hauts crée des barres 


\section{XI $I^{\text {èmes }}$ Journées Nationales Génie Côtier - Génie Civil}

Les Sables d'Olonne, 22-25 juin 2010

transverses (figure 4, cas 2) dite "regular" (SHORT \& AAGAARD, 1993). Quand la hauteur significative augmente, les liaisons à la côte se rompent pour former des barres festonnées bien marquées (figure 4, cas 1). Si l'incidence de la houle devient oblique, le motif festonné devient dissymétrique.

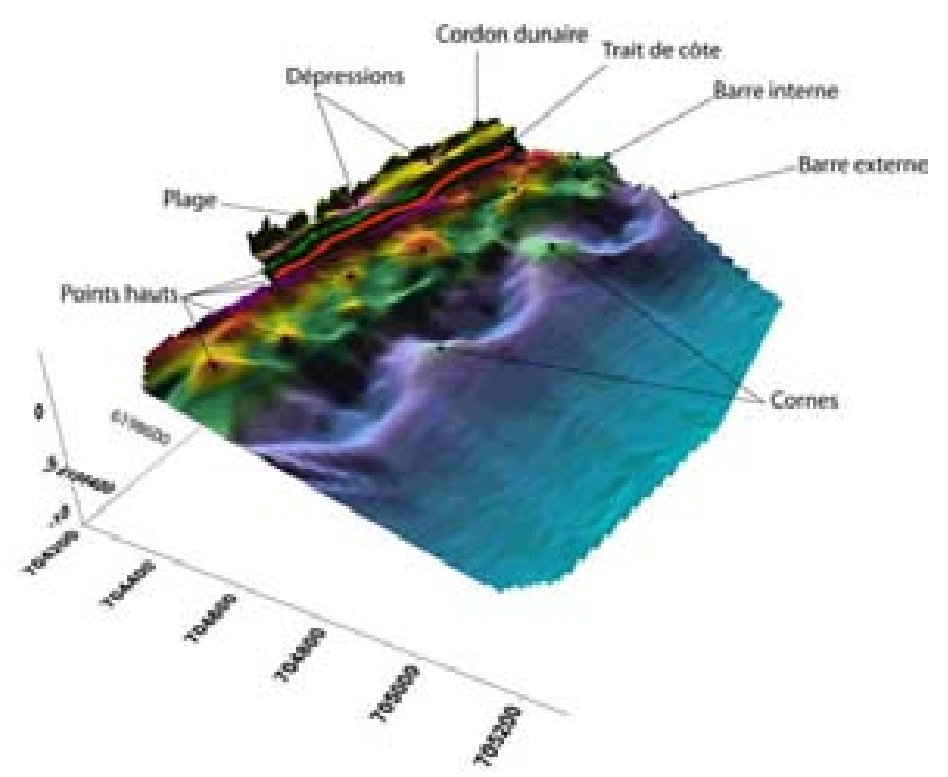

Figure 2. Carte bathymétrique tridimensionnelle de la zone d'étude montrant les barres festonnées et interne (en Lambert 93).
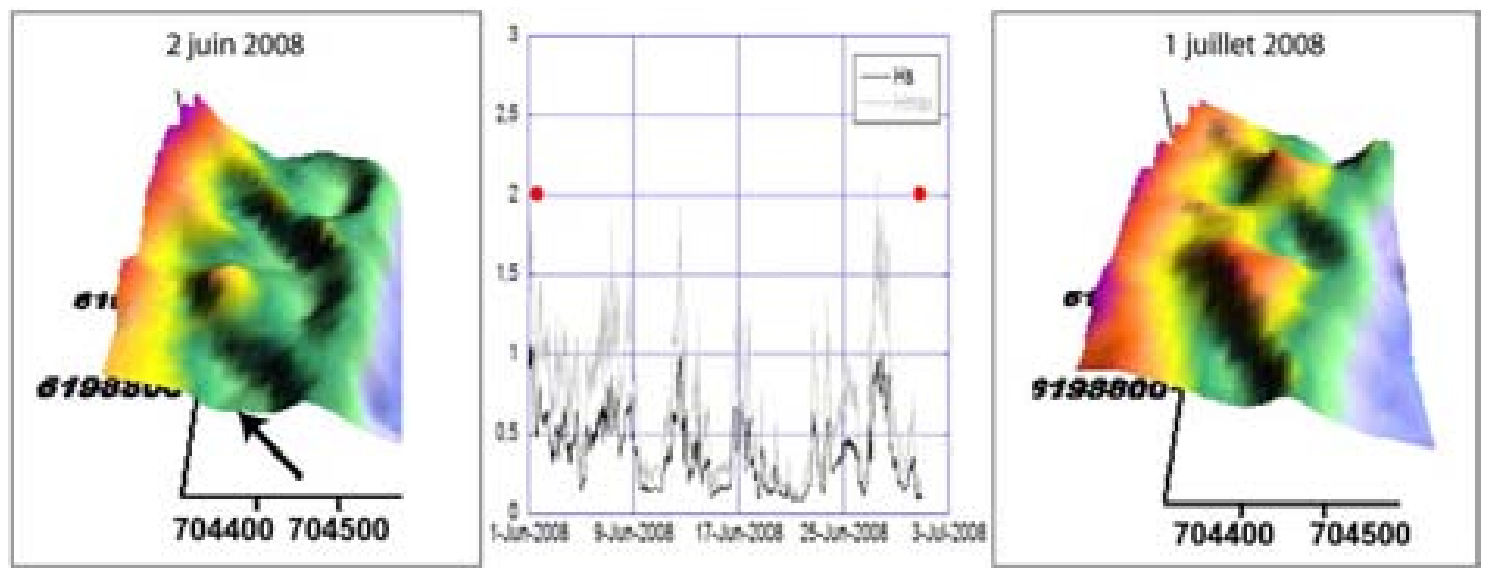

Figure 3. Evolution morphologique décrivant le passage d'un RBB dissymétrique à un TBR oblique pendant un évènement de faible énergie, $T$ varie entre $3 \mathrm{~s}$ et $8 \mathrm{~s}$ et l'incidence est oblique. La flèche noire indique l'orientation de la houle.

Si la configuration initiale est soumise à une houle d'incidence oblique, la crête du feston interne peut se rompre et former ainsi un nouvel état : RBB rompu (figure 4, cas 4). Lorsque les conditions énergétiques diminuent et qu'elles sont appliqués au système sur une longue période (de quelques semaines à un mois), les cornes des festons 
s'attachent à la côte formant des barres transverses obliques pendant que la crête du feston est toujours rompue. Le système prend alors une nouvelle configuration : TBR oblique rompu. Si le système est toujours soumis à des conditions de faible énergie et à une houle d'incidence oblique, les crêtes des festons se reconstruisent pendant que les cornes restent attachées à la côte, l'état ainsi obtenu est le TBR oblique (CASTELLE $e t$ $a l$. , 2007), (figure 4, cas 3 et 5). Cette configuration peut également être obtenue directement soit à partir de la configuration initiale en RBB sous l'action d'une houle faiblement énergétique et d'incidence oblique, soit à partir de l'état de RBB dissymétrique sous l'action d'une houle d'énergie décroissante et d'incidence oblique. Un dernier état peut être obtenu à partir des barres transverses obliques, celui de TBRLTT, utilisant des conditions de très faible énergie où le courant longitudinal domine (figure 4, cas 6). Cette configuration peut également être obtenue à partir de barres transverses dites "regular".

La configuration LBT, non observée durant le suivi, n'apparait pas dans notre modèle. Dans d'autres études, cet état a nécessité pour exister l'action de tempêtes décennales (CASTELLE et al., 2007) qui peuvent également entrainer la réinitialisation du système (SHAND et al., 2001).

\section{Discussion/Conclusion}

L'association des expérimentations de terrain réalisées entre 2000 et 2009 et des données hydrodynamiques disponibles a permis de mettre en évidence plusieurs configurations morphologiques pour le site de Leucate-Plage. Il apparait que la barre externe présente une configuration festonnée permanente, alors que la barre interne peut prendre plusieurs autres configurations comme TBR ou TBR-LTT.

Cette base de données a été utilisée pour élaborer un modèle conceptuel basé sur la classification de SHORT \& AAGAARD (1993). Pour être le plus précis possible dans la description, de nouveaux états ont été créés (RBB rompu et TBR rompu). L'enchainement de ces états s'explique par les variations de hauteur significative et l'incidence de la houle. Six changements morphologiques ont été observés et d'autres sont supposés possibles. Tous ces états observés en milieu microtidal peuvent être inclus dans les classifications existantes. Les séquences évolutives de la morphologie, décrites ici, montrent une bonne similarité avec celles observées et décrites pour des environnements microtidaux (GOLDSMITH et al., 1982). Outre la hauteur significative de la houle, son incidence est l'autre paramètre très important dans la morphodynamique des barres. Alors que ce modèle s'applique bien pour tous les systèmes microtidaux, son application pour les environnements méso- et macrotidaux est plus délicate. En présence d'une houle d'incidence frontale, les séquences évolutives sont assez similaires (DE MELO APOLUCENO, 2003; CASTELLE et al., 2007). Ces séquences donnent des informations sur la formation de l'état RBB, non observée ici. Lorsque la houle a une incidence oblique, la corrélation avec nos séquences évolutives 
se fait lors de la formation de RBB dissymétriques (SENECHAL et al., 2008), la formation de barres transverses obliques (CASTELLE et al., 2007), la transition de barres transverses vers un système TBR-LTT proche du système de barres/baïnes connus en Atlantique (DE MELO APOLUCENO, 2003 ; CASTELLE et al., 2007), mais également lors de la formation de petites structures en sortie de courant d'arrachement (BRANDER, 1999).

Les données de Leucate-Plage ont été acquises post-tempêtes. En dépit de la base de données conséquente, les états transitoires durant les évènements de haute énergie n'ont pas été observés. Le système semble donc très stable durant tout le suivi. Il serait intéressant d'associer un suivi vidéo au suivi topo-bathymétrique afin d'observer les changements syn-tempête des barres interne et externe. Cela permettrait certainement de vérifier la stabilité de la barre externe. Associé à cette technique, le marquage fluorescent des sédiments permettrait d'identifier et de quantifier la migration et les sources de sédiments.

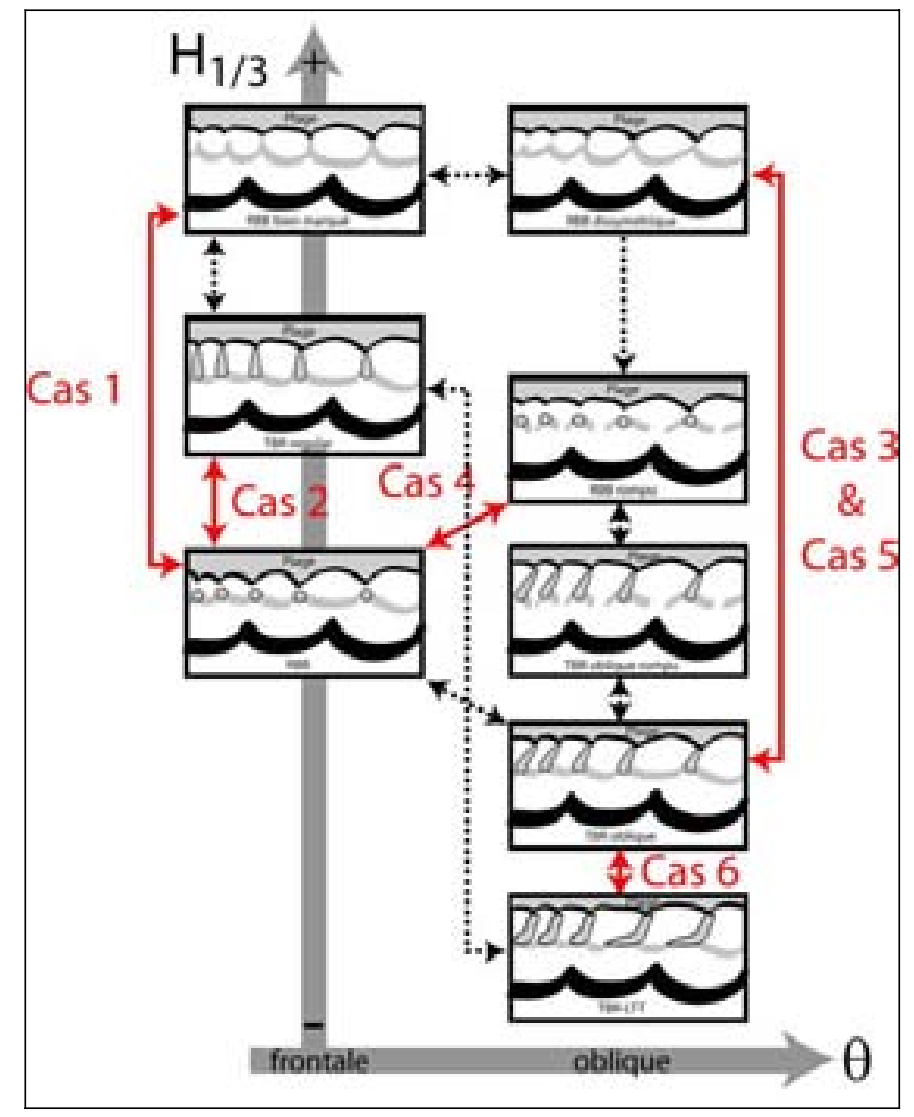

Figure 4. Modèle conceptuel morphodynamique pour le site de Leucate-Plage. $H_{1 / 3}$ st la hauteur significative de la houle avec une énergie croissante vers le haut et $\theta$ est l'incidence de la houle, soit frontale soit oblique. Les flèches en trait continu relient des états dont la transition a été observée, alors que celles en trait pointillé relient des transitions entre états probables supposés. 
Thème 2 - Dynamique sédimentaire et transports des particules

\section{Références bibliographiques}

BARUSSEAU J.P., SAINT-GUILY B. (1981). Disposition, caractères et formation des barres d'avant-côte festonnées du Golfe du Lion. Oceanologica Acta, 4, pp 297-304.

BRANDER R.W. (1999). Field observations on the morphodynamic evolution of a lowenergy rip current system. Marine Geology, 157, pp 199-217. doi:10.1016/S0025-3227(98)00152-2

CASTELlE B., BONNETON P., DUPUIS H., SENECHAL N. (2007). Double bar beach dynamics on the high-energy meso-macrotidal French Aquitanian Coast: A review. Marine Geology, 245, pp 141-159. doi:10.1016/j.margeo.2007.06.001

CERTAIN R. (2002). Morphodynamique d'une côte sableuse microtidale à barres: le golfe du Lion (Languedoc-Roussillon). Thèse, Université de Perpignan Via Domitia, France, 209 p.

CERTAIN R., BARUSSEAU J.P. (2005). Conceptual modeling of sand bars morphodynamics for a microtidal beach (Sète, France). Bulletin de la Société Géologique de France, $176: 343-354$. doi:10.2113/176.4.343

DE MELO APOLUCENO D. (2003). Morpho-hydrodynamique des plages à barres en domaine méso macro-tidal: exemple de la plage du Truc Vert, Gironde, France. Thèse, Université Bordeaux I, France.

FERRER P., CERTAIN R., BARUSSEAU J.P., GERVAIS M. (2009). Conceptual modelling of a double crescentic barred coast (Leucate Beach, France). Coastal Dynamics, Tokyo, Japan.

GOLDSMITH V., BOWMAN D., KILEY K. (1982). Sequential stage development of crescentic bars: Hahoterim Beach, Southeastern Mediterranean. Journal of Sedimentary Petrology, 52, pp 233-249.

RIVIERE A., ARBEY F., VERNHET S. (1961). Remarque sur l'évolution et l'origine des structures de plage à caractère périodique. C. R. Hebd. Seances Acad. Sci., 252, pp 767-769.

SENECHAL N., et al. (2008). ECORS - Truc Vert 2008 : Qualification des modèles de houle et de morphodynamique, $\mathrm{X}^{\text {èmes }}$ Journées Nationales Génie Côtier-Génie Civil, Sophia-Antipolis, France, pp 635-654. doi:10.5150/jngcgc.2008.061-S

SHAND R.D., BAILEY D.G., SHEPHERD M.J. (2001). Longshore realignment of shore-parallel sand-bars at Wanganui, New Zealand. Marine Geology, 179, pp 147161. doi:10.1016/s0025-3227(01)00223-7

SHORT A.D., AAGAARD T. (1993). Single and multi-bar beach change models. Journal of Coastal Research, Special Issue No. 15, pp 141-157.

VAN ENCKEVORT I.M.J., RUESSINK B.G., COCO G., SUZUKI K., TURNER I.L., PLANT N.G., HOLMAN R. (2004). Observations of nearshore crescentic sandbars. Journal of Geophysical Research, 109(C6), C06028. doi:10.1029/2003JC002214

WRIGHT L.D., SHORT A.D. (1984). Morphodynamic variability of surf zones and beaches: A synthesis. Marine Geology, 56, pp 93-118. doi:10.1016/0025-3227(84)90008-2 